\title{
Botulinum Toxin Type-A Treatment for Severe Trismus of Occlusal Origin Dominique Batifol ${ }^{1 *}$ and Béatrice Harding-Kaba ${ }^{2}$
}

${ }^{1}$ Department of Maxillofacial Surgery, Montpellier University Hospital, Montpellier, France

${ }^{2}$ Department of Maxillofacial Surgery, Cocody University Hospital, Abidjan, Ivory Coast

\section{Case Report}

A 65 year-old woman was referred to our department with limited mouth opening that was both extreme and painful and was causing difficulties with all facial expressions and making eating impossible. The dentist informed us that the patient believed the cause to be 'iatrogenic and prosthetic'. We were informed that the patient had changed odontologist and dental prostheses several times over the past 4 years.

In order to maintain the proportion of the lower third of the face, the size of the prosthetic teeth had been progressively increased. At the same time, the mouth opening had decreased to such an extent that, on the day of consultation, there was no mouth opening at all. Physical therapy had resulted in short-term improvements, but had proved ineffective over the longer term.

On the day of consultation, the patient was noted as being very thin, weighting just $48 \mathrm{~kg}$ and she reported having lost $10 \mathrm{~kg}$ in weight. Her facial expression appeared fixed and there was also a bilateral contraction of the masseter, temporal, sternocleidomastoid, buccinator, trapezius and platysma muscles. Endobuccal examination was very difficult because of the lack of mouth opening, however, a lot of wear was observed which was due to an off-centre bruxism occurring both night and day. The patient had dysfunctional swallowing and her attempts at mouth opening caused the simultaneous contraction of both agonist and antagonist muscles [1]. The patient had been taking anti-depressants for several months because her condition prevented her from being able to easily express herself and made eating very difficult, both of which had significantly negatively affected her mood.

Diagnosis of idiopathic muscular contractions was set. Differential diagnosis, either infectious as tetanus or dystonic as Meige's syndrome [2] cannot explain clinical observation seen in this case: indeed, there is no neurologic evidence for diagnosis of tetanus, and lacking of blepharospasma, familial reports or intermittent contractures allows to reject the hypothesis of a Meige's syndrome. The presentation was solely one of muscular contracture, without neurological signs, which drive to a new and unusual case.

The most appropriate treatment option was considered to be relaxation of the muscles concerned by injecting them with botulinum toxin type-A (BoNTA) (BOTOX ${ }^{\circ}$, Allergan Pharmaceuticals, Westport, Ireland) [3,4]. In addition, the prostheses were re-set using coronoplasty, thereby progressively reducing the vertical dimension, with a view to restoring the space between the arches while at rest. The temporary prostheses were adapted progressively to accommodate the patient's occlusal criteria requirements.

BoNTA was injected into the muscles under electromyographic control. A total dose of $250 \mathrm{U}$ Botox ${ }^{\star}$ was divided between specific muscles as follows: $30 \mathrm{U}$ per masseter, $10 \mathrm{U}$ per temporal, $30 \mathrm{U}$ per sternocleidomastoid, $10 \mathrm{U}$ per buccinator, $15 \mathrm{U}$ per platysma and $30 \mathrm{U}$ per trapezius. The first post-treatment follow-up consultation was scheduled 15 days later, during which it was noted that the injected muscles had relaxed, pain had clearly diminished and the mouth opening was now $19 \mathrm{~mm}$. The patient was able to eat more easily, although she was biting her cheeks.

Additional BoNTA injections were administered according to the same protocol 3 months later, since the beneficial effects of BoNTA are known to remain optimal for approximately 3 months [5]. At the follow-up consultation after the second series of injections the patient had gained $5 \mathrm{~kg}$ in weight, her mouth opening remained at $19 \mathrm{~mm}$, the muscles were relaxed and the subsequent improvement in her mood meant that antidepressants were now no longer required.

The patient now eats without difficulty and has increased weight to $59 \mathrm{~kg}$. Her mouth opening is $22 \mathrm{~mm}$, although this is not yet sufficient to allow complete prosthetic rehabilitation, but her general well-being has improved markedly. To date, the patient has received 4 sets of BoNTA injections at 3-monthly intervals and she has continued to improve throughout the treatment programme, although ongoing assessment is required since the muscle relaxation induced by BoNTA is temporary rather than permanent.

BoNTA is used in maxillo-facial surgery for treatment of much muscular and glandular pathology, including bruxism, facial paralysis, spasms and saliva hyper secretion [6]. For bruxism and temporomandibular joint dysfunctions, BoNTA is beneficial since it relaxes the elevator muscles in order to counteract the dysfunction, thereby protecting the joint and reducing pain [7]. In this particular case, it is rare to encounter such widespread contraction, which included all the cervical musculature and a large part of the facial musculature. Considerable damage can be caused by an error in prosthetic rehabilitation with consequences often far removed from the mouth cavity, but few patients lose so much weight and have such a complete closure of the mouth opening. BoNTA treatment was effective since it allowed the patient to return to a normal life and also enabled the odontologist to begin work on improving the patient's occlusion.

To our knowledge, the use of BoNTA in an occlusal pathology of this type has never before been reported. The results observed in this case are encouraging and will stimulate further interest in performing larger studies investigating the role of BoNTA for the treatment of manducator dysfunction characterised by an intense muscular tension with limited mouth opening following prosthetic rehabilitation.

*Corresponding author: Dominique Batifol, Department of Maxillofacial Surgery, Montpellier University Hospital, Montpellier, France, Tel: 0467338236 E-mail: dbpetronille13@gmail.com

Received January 16, 2014; Accepted March 24, 2014; Published March 27 , 2014

Citation: Batifol D, Harding-Kaba B (2014) Botulinum Toxin Type-A Treatment for Severe Trismus of Occlusal Origin. Anaplastology 3: 126. doi: 10.4172/21611173.1000126

Copyright: (C) 2014 Batifol D. This is an open-access article distributed under the terms of the Creative Commons Attribution License, which permits unrestricted use, distribution, and reproduction in any medium, provided the original author and source are credited. 
Citation: Batifol D, Harding-Kaba B (2014) Botulinum Toxin Type-A Treatment for Severe Trismus of Occlusal Origin. Anaplastology 3: 126. doi: 10.4172/2161-1173.1000126

\section{Financial Interests}

The authors have no financial interest in the use of the products reported in this paper.

\section{References}

1. Von Lindern JJ, Niederhagen B, Bergé S, Appel T (2003) Type A botulinum toxin in the treatment of chronic facial pain associated with masticatory hyperactivity. J Oral Maxillofac Surg 61: 774-778.

2. Tolosa E, Martí MJ (1988) Blepharospasm-oromandibular dystonia syndrome (Meige's syndrome): clinical aspects. Adv Neurol 49: 73-84.

3. Huang W, Foster JA, Rogachefsky AS (2000) Pharmacology of botulinum toxin. J Am Acad Dermatol 43: 249-259.
4. Spillane KS, Shelton JE, Hasty MF (2003) Stroke-induced trismus in a pediatric patient: long-term resolution with botulinum toxin A. Am J Phys Med Rehabil 82: 485-488.

5. Poulain B, Humeau $Y$ (2003) Mode of action of botulinum neurotoxin: pathological, cellular and molecular aspect. Ann Readapt Med Phys 46: 265275 .

6. Sycha T, Kranz G, Auff E, Schnider P (2004) Botulinum toxin in the treatment of rare head and neck pain syndromes: a systematic review of the literature. $J$ Neurol 251: 119-30.

7. Chikhani L, Dichamp J (2003) [Bruxism, temporo-mandibular dysfunction and botulinum toxin]. Ann Readapt Med Phys 46: 333-337. 